\title{
Elaboração de telas eletrônicas em um sistema de informação hospitalar utilizando as classificações de enfermagem*
}

Creating electronic screens in a hospital information system using nursing classifications

Elaboración de pantallas electrónicas en un sistema informático hospitalario utilizando las clasificaciones de enfermería

Dorothea Maria Beckers Marques de Almeida ${ }^{1}$, Cassiana Mendes Bertoncello Fontes ${ }^{2}$

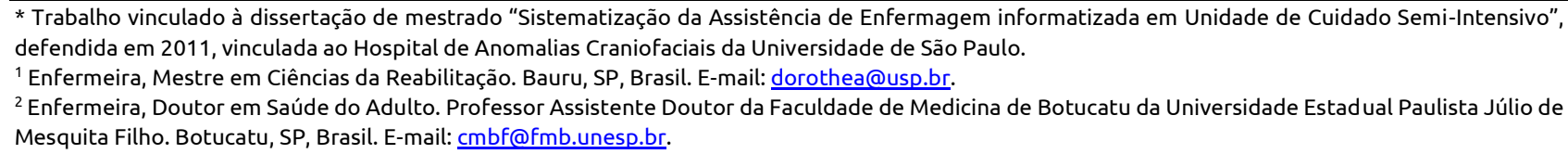

\section{RESUMO}

O registro eletrônico tem a finalidade de proporcionar maior legibilidade e segurança na documentação das ações do enfermeiro. A utilização das classificações de enfermagem inseridas em um sistema informatizado organiza, uniformiza e resgata os dados essenciais para o planejamento do cuidado individualizado e a avaliação dos resultados advindos das intervenções. O objetivo foi descrever a elaboração das etapas da Sistematização da Assistência de Enfermagem (SAE) utilizando as Classificações de Enfermagem em um Sistema de Informação Hospitalar (SIH). Um mapeamento cruzado entre o seu conteúdo e o impresso do plano de cuidados da Unidade de Cuidado Semi-Intensiva foi realizado. Planilhas em Excel foram inseridas de acordo com as etapas da sistematização e telas eletrônicas permitiram o registro da avaliação clínica do enfermeiro. A documentação eletrônica proporcionou agilizar e resgatar dados, estimular a acurácia diagnóstica e avaliar as intervenções por meio dos indicadores de resultados.

Descritores: Diagnóstico de Enfermagem; Processos de Enfermagem; Assistência de Enfermagem; Informática em Enfermagem.

\section{ABSTRACT}

Electronic health records aim at providing greater readability and safety in documenting nursing actions. Using the nursing classifications of an information system organizes, standardizes and retrieves the data considered essential to plan individualized care and the results evaluation from the interventions. The objective was to describe the development of the Nursing Care Systematization stages using Nursing Classifications in a Hospital Information System. Cross-mapping was performed of the care plan content and the printed version, at a Semi-Intensive Care Unit. Excel spreadsheets were included according to the systematization stage and electronic screens permitted to record the nurses' clinical evaluation. The electronic documentation permitted faster data input and retrieval, in addition to stimulating diagnosis accuracy and evaluating the interventions by means of result indicators.

Descriptors: Nursing Diagnosis; Nursing Process; Nursing Care; Nursing Informatics.

\section{RESUMEN}

El registro electrónico tiene la finalidad de proporcionar mayor legibilidad y seguridad a la documentación de las acciones del enfermero. La utilización de las clasificaciones de enfermería insertas en un sistema informático organiza, otorga uniformidad y resguarda los datos esenciales para la planificación del cuidado individualizado y la evaluación de los resultados derivados de las intervenciones. Se objetivó describir la elaboración de las etapas de la SAE utilizando las Clasificaciones de Enfermería en un Sistema Informático Hospitalario (SIH). Se realizó un mapeo cruzado entre su contenido y la versión impresa del plan de cuidados de la Unidad de Terapia Intermedia. Fueron incluidas planillas de Excel de acuerdo con las etapas de la sistematización, y las pantallas electrónicas permitieron registrar la evaluación clínica del enfermero. La documentación electrónica permitió agilizar y resguardar datos, estimular la precisión diagnóstica y evaluar las intervenciones mediante los indicadores de resultados.

Descriptores: Diagnóstico de Enfermería; Procesos de Enfermería; Atención de Enfermería; Informática Aplicada a la Enfermería. 


\section{INTRODUÇÃO}

A Sistematização da Assistência de Enfermagem (SAE) é constituída por etapas inter-relacionadas: investigação, diagnóstico, planejamento, implementação e avaliação de enfermagem. Isto pode proporcionar instrumentalização das ações do enfermeiro; porém, sua documentação demanda tempo e produz impacto na carga de trabalho(1).

O registro eletrônico da SAE vem sendo desenvolvido com a finalidade de proporcionar ao enfermeiro maior legibilidade e segurança no armazenamento e organização dos dados, informação e conhecimento para a tomada de decisão, beneficiar o gerenciamento das atividades da enfermagem, avaliar o custo/benefício do cuidado prestado, melhorar a produtividade e a qualidade da assistência prestada, bem como otimizar o tempo dispensado para o planejamento do cuidado $^{(2-5)}$.

As classificações de enfermagem inseridas em um sistema eletrônico contribuem como forma de linguagem padronizada e comum a ser utilizada universalmente pelos profissionais no planejamento da assistência, ensino e pesquisa ${ }^{(6-7)}$.

Na prática clínica ${ }^{(8)}$ evidenciou-se um benefício na utilização de linguagens padronizadas. A implementação de um projeto de informatização de registro dos dados clínicos de enfermagem oportunizou o entrosamento e entusiasmo da equipe, dos professores, alunos de graduação e pesquisadores, pois o conteúdo curricular contemplava as terminologias das Classificações de Intervenções e Resultados de Enfermagem.

O Diagnóstico de Enfermagem (DE) é um "julgamento clínico das respostas do indivíduo, da família ou da comunidade a problemas de saúde/processos vitais, reais ou potenciais, e constitui a base para a seleção das intervenções de enfermagem e o alcance dos resultados pelos quais o enfermeiro é responsável"(9).

As Intervenções de Enfermagem (IE) são compostas por atividades que podem ser implementadas a partir dos Resultados de Enfermagem (RE) esperados para o DE nomeado pelo enfermeiro. Os RE possuem Indicadores que permitem mensurar o grau de eficiência da IE proposta ${ }^{(10-11)}$.

As classificações de enfermagem NANDA-I(9) (North American Nursing Diagnosis Association), NIC(10) (Nursing Interventions Classification) e $\operatorname{NOC}^{(11)}$ (Nursing Outcomes Classification), inseridas em um sistema eletrônico, fornecem informações essenciais, integra, organiza e melhora a qualidade e a documentação dos cuidados de enfermagem, encoraja os enfermeiros a adotarem a SAE. Isso aprimora a acurácia diagnóstica, proporciona resolução individualizada e alcance dos resultados obtidos junto aos pacientes, além de contemplar uniformização à continuidade dos dados, qualificar o cuidado e avaliar a eficiência da enfermagem ${ }^{(5,12-15)}$.

Os Sistemas de Informação e a informática são recursos disponíveis que podem aprimorar, organizar, fortalecer o cuidado de Enfermagem, gerenciar indicadores da assistência de enfermagem, fornecendo, em tempo real, todo e qualquer dado que o enfermeiro necessita para o desenvolvimento de suas ações ${ }^{(16-17)}$.

Esse estudo propõe um modelo de registro eletrônico da SAE, utilizando as classificações de enfermagem. Atualmente a SAE é registrada manualmente no prontuário do paciente em todas as unidades do Hospital de Reabilitação de Anomalias Craniofaciais da Universidade de São Paulo (HRAC-USP).

A implementação da SAE na Unidade de Terapia SemiIntensiva (UTSI), local do estudo, iniciou-se em 1987 com as etapas de histórico, evolução e prescrição de enfermagem. Essa unidade tem como finalidade investigar, tratar e reabilitar crianças até dois anos de idade com fissura labiopalatina associada ou não à anomalia craniofacial e problemas que abrangem a disfagia, obstrução de vias aéreas superiores, refluxo gastroesofágico e alterações neurológicas. A fase do DE foi implementada em 2004, a partir da identificação do perfil das necessidades de cuidados, segundo referencial da NANDA.

O registro das etapas da SAE de forma manuscrita no prontuário do paciente consome tempo do enfermeiro, que pode interferir na qualidade da assistência. A tecnologia da informação e as classificações de enfermagem podem otimizar esse processo de trabalho e assim o enfermeiro despenderá mais tempo na assistência direta ao paciente.

O objetivo desse estudo foi descrever a elaboração das etapas da SAE utilizando as Classificações de Enfermagem em um Sistema de Informação Hospitalar (SIH). Acredita-se que esse estudo poderá encorajar os enfermeiros a utilizarem o registro eletrônico como ferramenta da SAE. 


\section{MÉTODO}

Estudo descritivo foi realizado no Hospital de Reabilitação de Anomalias Craniofaciais da Universidade de São Paulo (HRAC-USP). O local de estudo foi a Unidade de Terapia Semi-Intensiva (UTSI), composta por oito leitos destinados à internação de lactentes e crianças até dois anos de idade, com fissura labiopalatina associada ou não à anomalia craniofacial. A SAE é realizada diariamente por registro manual. A amostra consistiu de 13 pacientes avaliados por 10 dias consecutivos. Essa avaliação inclui exame físico de enfermagem, observação dos registros no prontuário de cada paciente, inserção dos dados de interesse clínico e elaboração do plano de cuidados nas telas no sistema "Gandhi".

O SIH utiliza o módulo computacional denominado Sistema "Gandhi" como sistema gerenciador de banco de dados, em linguagem de programação PROGRESS - 4GL ${ }^{\circledR}$, contendo informações relacionadas aos processos de produção de trabalhos administrativos e assistenciais do paciente. Рага o acesso ao sistema, efetua-se login e senha.

As etapas da SAE eletrônica, em primeiro momento, constituíram-se na elaboração de planilhas em formato Excel com o banco de dados de DE, IE, atividades, RE e indicadores. Em relação aos $D E$ foi realizado um mapeamento cruzado entre aqueles DE que constavam no impresso do plano de cuidados da unidade(18) com a versão 2009-2011 da NANDA-I(9). A proposição das IE/Atividades e RE/Indicadores foram embasadas na prática clínica das ações de enfermagem dispensadas aos pacientes da unidade. Foram preservados os respectivos códigos numéricos de acordo com NIC(10) e $\mathrm{NOC}^{(11)}$. Em segundo momento, as planilhas, os DE, IE e RE foram inseridas no Sistema "Gandhi" pela equipe do SIH e as telas foram organizadas e nomeadas.

O projeto desse estudo foi aprovado pelo Comitê de Ética em Pesquisa, ofício número 264/2011, com dispensa do TCLE. A pesquisa foi restrita à descrição do uso das telas eletrônicas e não da intervenção com pessoas.

\section{RESULTADOS}

A inserção dos dados em planilhas no formato Excel no SIH, módulo computacional Sistema "Gandhi", proporcionou o desenvolvimento da SAE eletrônica constituída de oito funcionalidades: SAE - Diagnósticos; SAE - Intervenções; SAE - Atividades; SAE - Resultados;
SAE - Indicadores; SAE - Diagnósticos x Intervenções; SAE - Atividades Indicadores; SAE - Prescrição ao paciente. Para efetuar login, necessita-se de usuário e senha.

As funcionalidades da SAE permitem realizar manutenção cadastral, adicionar, excluir ou alterar dados. Para qualquer operação, os ícones de salvar e excluir devem ser selecionados e os dados salvos são redirecionados para a tela "SAE- prescrição ao paciente", última do sistema.

O banco de dados em planilhas Excel foi utilizado para preparar o conteúdo das telas de cadastro e consulta de: diagnósticos, intervenções, resultados, indicadores e inter-relacioná-las.

A SAE eletrônica inserida no SIH foi organizada conforme as fases a serem elaboradas pelo usuário, desde avaliação do enfermeiro com exame físico, evolução, julgamento clínico dos $D E$, seleção das atividades, IE e respectivos indicadores e RE. A tela "SAE- Cadastro de Diagnóstico" permite ao enfermeiro consultar a relação dos DE e selecionar de acordo com sua avaliação clínica (Figura 1). Esse procedimento direciona para a tela "SAE Intervenções" (Figura 2). 
Figura 1: Tela de consulta dos Diagnósticos. HRAC-USP, Bauru, SP, Brasil, 2010.

\begin{tabular}{|c|c|c|c|}
\hline Consulta & dos Diagnósticos - SAE & & $\mathrm{x}$ \\
\hline Versao & \begin{tabular}{|l|l|} 
Diagnostico & Descricao \\
\end{tabular} & & $\Delta$ \\
\hline 2009.01 & 0001 Risco de infecção & & \\
\hline 2009.01 & 0002 Risco de aspiração & & \\
\hline 2009.01 & 0003 Mucosa oral prejudicada & & \\
\hline 2009.01 & 0004 Integridade da pele prejudicada & & \\
\hline 200901 & 000 'Deglutição prejudicada & & \\
\hline 2009.01 & 0006 Integridade tissular prejudicada & & \\
\hline 2009.01 & 0007 Padrão respiratório ineficaz & & \\
\hline 2009.01 & 0008 Desobstrução ineficaz de vias aéreas & & \\
\hline 2009.01 & 0009 Padrão ineficaz de alimentação infantil / bebễ & & \\
\hline 2009.01 & 0010 Déficit no autocuidado & & \\
\hline 2009.01 & 0011 Desempenho de papel ineficaz & & \\
\hline 2009.01 & 0012 Enfrentamento do cuidador ineficaz & & \\
\hline 2009.01 & 0013 Enfrentamento familiar comprometido & & \\
\hline 1 & & D & \\
\hline
\end{tabular}

Figura 2: Tela de consulta de Intervenções. HRAC-USP, Bauru, SP, Brasil, 2010.

\begin{tabular}{|c|c|c|}
\hline Consulta & das Intervençóes - SAE & \\
\hline Versao & \begin{tabular}{|l|l|} 
Intervencao & Descricao
\end{tabular} & $\Delta$ \\
\hline 2009.01 & 1803 Assistência no autocuidado: alimentação & \\
\hline 2009.01 & 1860 Terapia para deglutição & \\
\hline 2009.01 & 1875 Cuidados com cateter: linha umbilical & \\
\hline 2009.01 & 2300 Administração de medicamentos & \\
\hline 2009.01 & 2311 Administração de medicamentos: inalação & \\
\hline 2009.01 & 3120 Inserção e estabilização de vias aéreas artificiais & \\
\hline 2009.01 & 3140 Controle de vias aéreas & \\
\hline 2009.01 & 3160 Aspiração de vias aéreas ............... & \\
\hline 2009.01 & 3200 Precauções/prevenção contra aspiração & \\
\hline 2009.01 & 3320 Oxigenoterapia & \\
\hline 2009.01 & 3350 Monitoração respiratória & \\
\hline 2009.01 & 3390 Assistência ventilatória & $\nabla$ \\
\hline
\end{tabular}

A Figura 2 demonstra a "tela de consulta de Intervenções", acesso para selecionar as intervenções de acordo com o DE nomeado.

A tela "consulta das Atividades das Intervenções" (Figura 3) demonstra a relação de intervenções e atividades possíveis de serem escolhidas, a partir dos DE selecionados na "tela de consulta dos Diagnósticos". Essa interface ocorre a partir da tela "SAE - Atividades" e é elaborada para cada DE. O conteúdo selecionado, após confirmação no ícone "confirmar esse diagnóstico com essas atividades", é redirecionado para a tela com as
atividades/IE relacionadas a cada DE com o seu respectivo indicador de resultado. 
Figura 3: Tela de consulta das Atividades das Intervenções. HRAC-USP, Bauru, SP, Brasil, 2010.

SAE - Consulta das Atividades das Intervenções

\begin{tabular}{|c|c|c|c|}
\hline Versao & \begin{tabular}{|l|l|} 
Intervencao Descricao \\
\end{tabular} & Atividade Descricao & 스 \\
\hline 2009.01 & 3160 Aspiração de vias aéreas & 316001 Determinar a necessidade de aspiraçõo oral e/ou trax & \\
\hline 2009.01 & 3160 Aspiração de vias aéreas & 316002 Auscultar os sons respiratórios antes e após a aspiraç & \\
\hline 2009.01 & 3160 Aspiração de vias aéreas & 316003 Informar o paciente e a família sobre a aspiração & \\
\hline 2009.01 & 3160 Aspiração de vias aéreas & 316004 Aspirar a nasofaringe com dispositivo de aspiração, c & \\
\hline 2009.01 & 3160 Aspiração de vias aéreas & 316005 Usar precauções padronizadas: luvas, óculos e másc & \\
\hline 2009.01 & 3160 Aspiração de vias aéreas & 316006 Inserir dispositivo nasal para facilitar a aspiração nasc & \\
\hline 2009.01 & 3160 Aspiração de vias aéreas & 316007 Usar equipamento esterilizado descartável para cada & \\
\hline 2009.01 & 3160 Aspiração de vias aéreas & 316008 Monitorar o estado de oxigenação do paciente [nivei & \\
\hline 2009.01 & 3160 Aspiração de vias aéreas & 316009 Aspirar a orofaringe após o término da aspiração da tr & \\
\hline 2009.01 & 3160 Aspiração de vias aéreas & 316010 Limpar a área em torno do estoma traqueal após o tér & \\
\hline 2009.01 & 3160 Aspiração de vias aéreas & 316011 Orientar cuidador sobre forma de aspirar as vias aére: & \\
\hline 2009.01 & 3160 Aspiração de vias aéreas & 316012 Hiperoxigenar com oxigênio a $100 \%$, usando ventilad, & \\
\hline
\end{tabular}

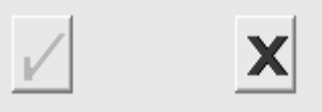

A tela "SAE - Atividades x Indicadores" proporciona a seleção de Indicador do resultado desejado e seu respectivo score (Figura 4), possibilitando ao usuário excluir e adicionar a atividade e a intervenção respectivamente por meio dos ícones "excluir a relação indicador $x$ atividade selecionada acima" e "adicionar este indicador para a atividade acima". 
Figura 4: Tela de cadastro dos Indicadores de Resultados e das

Atividades das Intervenções. HRAC-USP, Bauru, SP, Brasil, 2010.

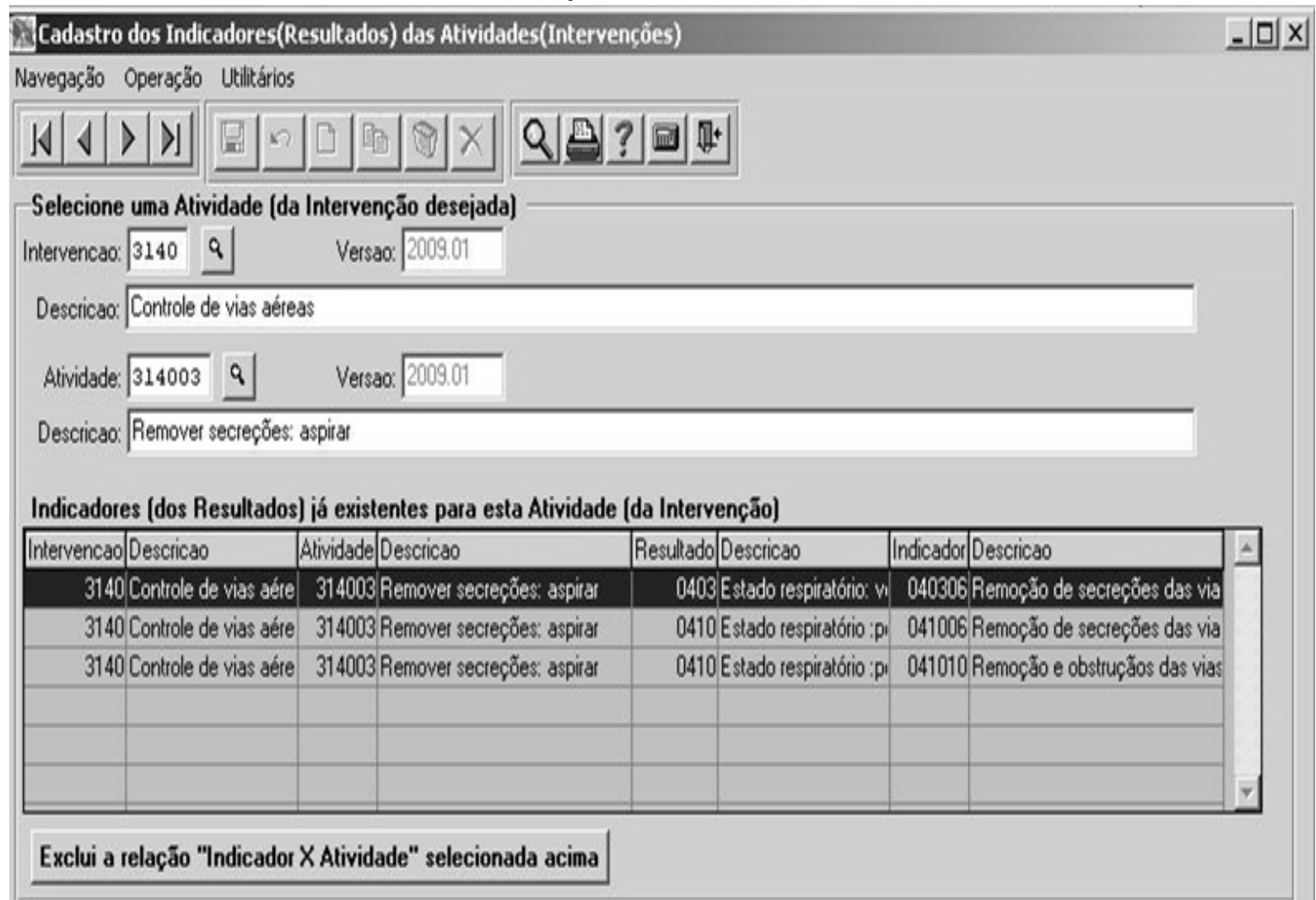

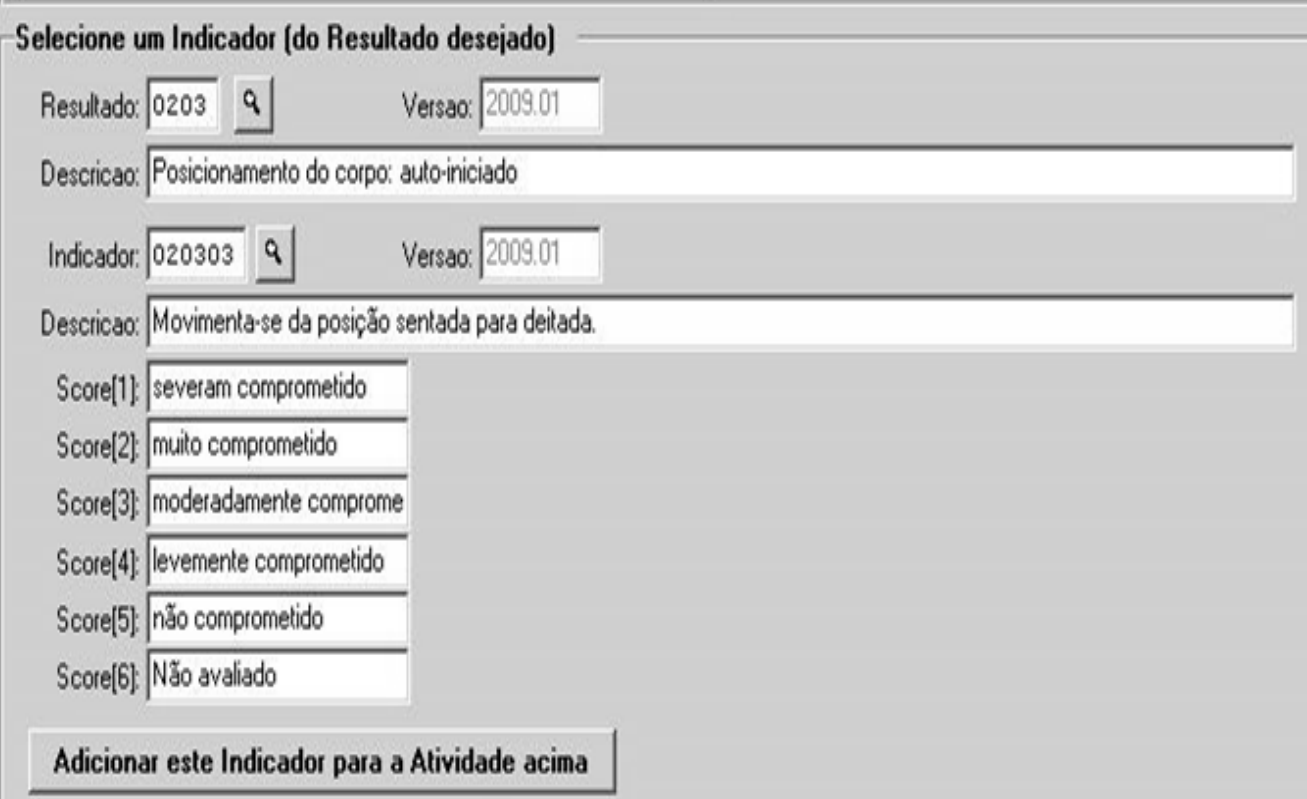

A Figura 5 representa a tela do registro dos dados clínicos. Por meio da funcionalidade menu "SAE Prescrição ao paciente" e do registro geral do paciente, o sistema fornece nome, idade, histórico das internações anteriores e atual, tipo de tratamento e condição atual. $O$ ícone "Nova PRESCRIÇÃO" direciona para a tela de inserção dos dados da evolução de enfermagem em caixa de texto. 
Figura 5: Tela Sistematização da Assistência de Enfermagem - Prescrição ao paciente com os dados nomeados. HRACUSP, Bauru, SP, Brasil, 2010.

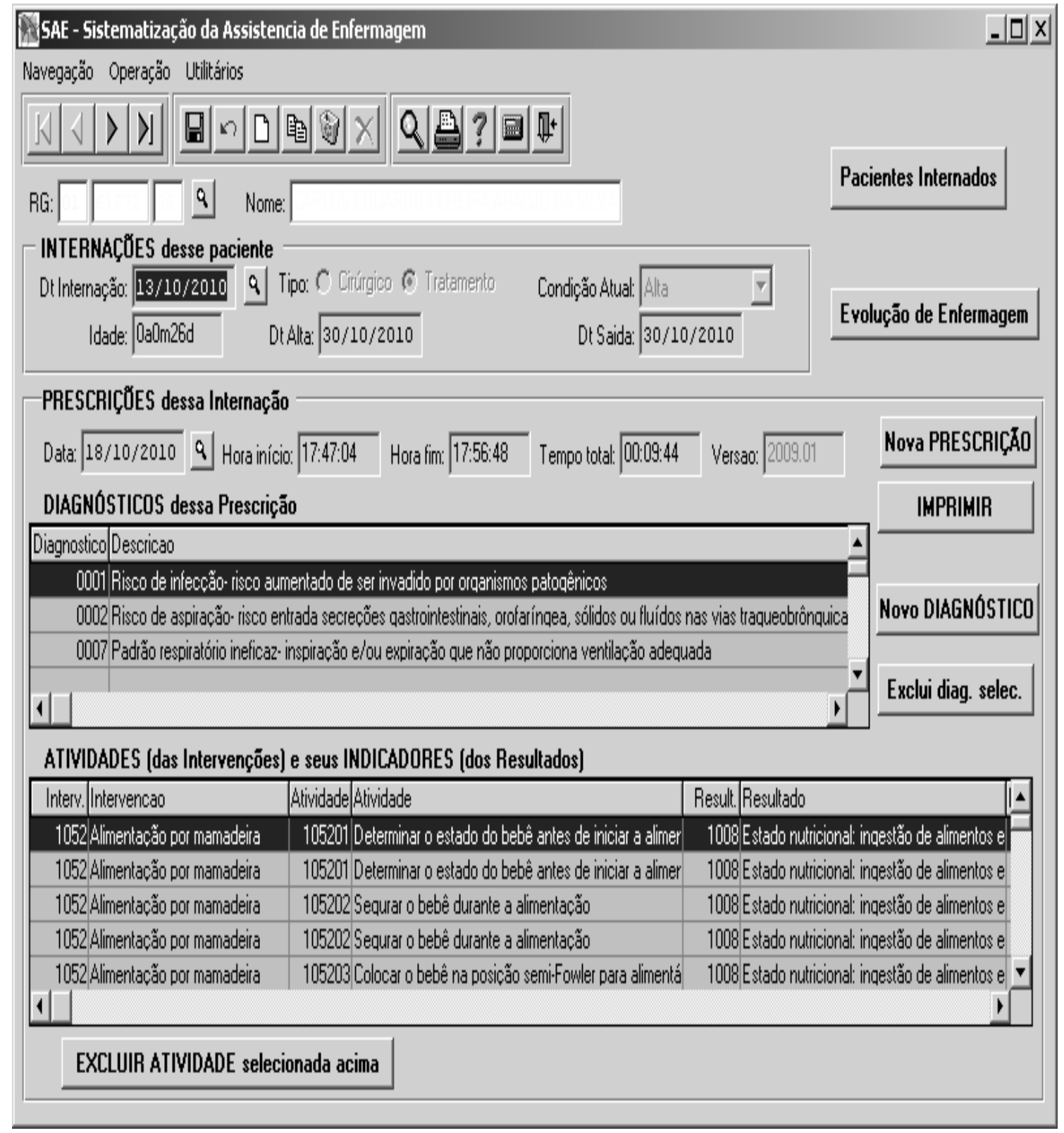

Essa tela é a última funcionalidade que pode ser visualizada e impressa. O sistema permite: uma nova evolução diária ou, quando necessária, clicando em “Evolução de Enfermagem”, acrescentar DE em "Novo Diagnóstico", mantendo os registros anteriores para consulta.

\section{DISCUSSÃO}

A utilização do conteúdo das classificações da NANDA-I, NIC, NOC como referenciais foi o primeiro passo para a documentação eletrônica de enfermagem no HRAC-USP.

Podemos verificar que, em estudo em hospital universitário o tempo despendido pela enfermeira na realização da SAE após a implementação da classificação da NANDA contribuiu para um maior tempo do cuidado direto ao paciente e proporcionou um leque mais amplo dos fenômenos a serem tratados pela enfermagem ${ }^{(19)}$. Em outro hospital de ensino, uma documentação eletrônica de enfermagem foi desenvolvida em quatro fases e organizada em hierarquia de domínios e classes, da NANDA-I, NIC e NOC. Isso contribuiu para a gestão da informação e a sensibilização dos enfermeiros na informatização da documentação clínica ${ }^{(14)}$.

O formato eletrônico proporcionou uma visualização do planejamento de enfermagem com base no julgamento clínico do enfermeiro que demonstrou as possibilidades de selecionar as ações apropriadas para 
cada DE eleito. A definição dos indicadores de resultados colabora para a avaliação do cuidado a ser implementado.

Essas classificações são instrumentos que contribuem para melhorar a confiabilidade, a validade e a usabilidade da documentação de enfermagem. Permite ao enfermeiro pesquisar, consultar, trocar informações e experiências, resgatando os dados clínicos de interesse ${ }^{(14)}$.

Estudo que compara as intervenções de enfermagem desenvolvidas por enfermeiros assistenciais com aquelas preconizadas pela NIC considera que intervenções prioritárias auxiliam os enfermeiros assistenciais a desenvolver planos de cuidados específicos com resolutividade(20).

A preservação dos códigos numéricos originais da NIC e NOC para as IE/Atividades e dos RE/Indicadores colaboram para uma análise das frequências de suas ocorrências. Poderão ser utilizados, também, como indicadores de avaliação em pesquisas clínicas que visem demonstrar o perfil de necessidades de intervenções. $O$ dimensionamento da carga de trabalho de enfermagem poderá também receber contribuição, pois o resgate eletrônico dos dados possibilitará visualização da quantidade de intervenções e atividades para uma população específica.

A articulação consistente das etapas da SAE, inseridas em um sistema eletrônico, utilizando as linguagens padronizadas, pode oferecer eficiência, fornecer agrupamentos de diagnósticos, intervenções e resultados, contribuir para a avaliação da assistência e direcionar as decisões de natureza clínica e gerencial( $(7,14,16,21)$.

Um Sistema de Informação Hospitalar é considerado como ferramenta facilitadora não só do trabalho administrativo, mas também na prática das ações de enfermagem por meio do planejamento da assistência, manuseio e acesso aos dados ${ }^{(3-4,14)}$. A tecnologia no

\section{REFERÊNCIAS}

1. Perroca MG. Desenvolvimento e validação de conteúdo da nova versão de um instrumento para classificação de pacientes. Rev Latino Am Enferm. 2011;19(1):58-66.

2. Lunney M. The need for international diagnosis research and a theoretical framework. Int J Nurs Terminol Classif. 2008;19(1):28-34.

3. Chianca TCM, Souza CC, Werli A, Hamze FL, Ercole FF. Uso das intervenções de enfermagem na prática clínica no Brasil. Rev. processo de trabalho em saúde representa a melhoria da prática assistencial(22).

A apropriação do conhecimento advindo de terminologias de sistemas de classificação para o cuidado em saúde, especificamente em enfermagem, é importante para assegurar a qualidade e segurança do cuidado. É um grande desafio aliar sistemas de classificação com os recursos tecnológicos; porém, favorecerá o registro eletrônico dos dados clínicos. A SAE eletrônica proporcionará a comunicação entre os pares e, o processo de tomada de decisão define o cuidado eficaz, qualificado e seguro, contribuindo para o desenvolvimento, atualização e disseminação de novas experiências e conhecimentos ${ }^{(16)}$.

\section{CONCLUSÃO}

A SAE eletrônica foi composta por oito telas e suas funcionalidades. A introdução do conteúdo das classificações de enfermagem da NANDA, NIC e NOC foi um grande desafio e uma possibilidade de visualização dos conteúdos inter-relacionados. A disposição em tempo real do sistema eletrônico e as opções de inserir, excluir, visualizar e resgatar os dados clínicos de interesse proporcionou visibilidade do processo de planejamento do cuidado.

A padronização da linguagem por meio das ligações NANDA-I, NIC e NOC, associada a um sistema eletrônico de dados clínicos de paciente certamente acelera o conhecimento na enfermagem e constitui um importante instrumento na prática da enfermagem baseada em evidências, na educação, ensino e pesquisa.

O estudo demonstrou viabilidade de futura implementação da SAE eletrônica e poderá trazer o desenvolvimento de novos DE, IE e RE, pois armazenará em um banco de dados clínicos gerado pelo registro eletrônico. Isso é um subsídio da prática, para o ensino e a pesquisa de enfermagem, em estudos de perfil das necessidades de cuidados, de intervenções e resultados.

Eletr. Enf. [Internet]. 2009 [acesso em: $20 \mathrm{dez}$ 2013];11(3):47783. Disponível em:

http://www.fen.ufg.br/revista/v11/n3/v11n3a03.htm.

4. Anjos KF, Santos VC, Almeida OS, Simão AAG. Implementação informatizada da Sistematização da assistência de enfermagem: uma proposta na evolução do cuidar. ConScientia e saúde [Internet]. 2010 [cited 2012 Set 7];9(1):147-154. Disponível em: http://www4.uninove.br/ojs/index.php/saude/article/viewFile/2 $144 / 1701$. 
5. Dal Sasso GTM, Barra DCC, Paese F, Almeida SRW, Rios GC, Marinho MM, et al. Processo de enfermagem informatizado: metodologia para associação da avaliação clínica, diagnósticos, intervenções e resultados. Revista da Escola de Enfermagem da USP. [Internet]. 2013 [cited 2013 out 15];47(1):242-9. Disponivel em: http://www.scielo.br/pdf/reeusp/v47n1/a31v47n1.pdf 6. Cruz D de ALM da. Processo de enfermagem e classificações. In: Gaidzinski RR, Soares AVN, Lima AFC, Gutierrez BAO, Cruz D de ALM da, Rogenski NMB, et al. Diagnóstico de enfermagem na prática clínica. Porto Alegre: Artmed; 2008. p.25-37.

7. Sistematização da Assistência de Enfermagem informatizada em Unidade de Cuidado Semi-Intensivo [dissertação]. Bauru: Hospital de Anomalias Craniofaciais, Universidade de São Paulo; 2011.

8. Hendrix SE. An experience with implementation of NIC and $\mathrm{NOC}$ in a Clinical Information System. CIN:Computers, Informatics, Nursing.January/February. 2009;7-11. 9. North American Nursing Diagnosis Association. Diagnósticos de Enfermagem da NANDA: definições e classificação 20092011. NANDA Internacional. Trad. RM Garcez. Porto Alegre: Artmed; 2010.

10. Bulechek GM, Butcher HK, Dochterman JM. Classificações das intervenções de enfermagem NIC (Tradução Soraya Imon de Oliveira et al). Rio de Janeiro: Elsevier, 2010.

11. Moorhead S, Johnson M, Maas ML, Swanson E. Classificação dos resultados de enfermagem - NOC. (tradução de Regina Machado Garcez et al). Rio de Janeiro, Elsevier, 2010.

12. Lunney M. Helping nurses use NANDA, NOC and NIC. Nurse Educator. 2006:31(1):40-46.

13. Von Krogh G, Nåden D. A nursing-specific model of EPR documentation: organizational and professional requirements. J Nurs Sholarsh. 2008;40(1):68-75.

14. Peres HHC, Cruz DALM, Lima AFC, Gaidzinski RR, Ortiz DCF, Trindade MM, et al. Desenvolvimento de sistema eletrônico de documentação clínica de enfermagem estruturado em diagnósticos, resultados e intervenções. Revista da Escola de Enfermagem da USP. 2009; 43(Esp 2):1149-55.

15. Pereira JC, Stuchi RAG, Arreguy-Sena C. Proposta de sistematização da assistência de enfermagem pelas taxonomias nanda/nic/noc para o diagnóstico de conhecimento deficiente. Cogitare enferm. 2010;15(1):74-81.

16. Barra DCC, Sasso GTMD. Padrões de dados, terminologias e sistemas de classificação para o cuidado em saúde e enfermagem. Rev. bras. enferm. Brasilia. 2011;64(6):1141-9. 17. Labbadia LL, D'Innocenzo M; Fogliano RRF, Silva GEF et al. Sistema Informatizado para Gerenciamento de Indicadores da Assistência de Enfermagem do Hospital São Paulo. Revista da Escola de Enfermagem da USP. 2011;45(4):1013-17.

18. Fontes $C M B$, Bertone $A M C$, Sassaki $C H$. Implementação da fase do diagnóstico de enfermagem nos registros de enfermagem da unidade de terapia intensiva do Hospital de Reabilitação de Anomalias Craniofaciais. [Pôster]. Encontro Internacional de Disfagia Pediátrica, $4^{\circ}$, Encontro Nacional em Disfagia Pediátrica, $3^{\circ} ; 2006$, out.25-28; Bauru: Hospital de Reabilitação de Anomalias Craniofaciais.

19. Rezende PO, Gaidzinski RR. Tempo despendido no sistema de assistência de enfermagem após implementação de sistema padronizado de linguagem. Revista da Escola de Enfermagem da USP. 2008;42(1):152-9.

20. Monteiro FPM, Silva VM da, Lopes MV de O, Araújo TL de. Desobstrução ineficaz das vias aéreas: mapeamento das atividades de enfermagem para crianças com infecção respiratória. Rev Eletrônica Enferm. [Internet]. 2008 [cited 2012 set 7];10(3):563-72.

Disponivel em:

http://www.fen.ufg.br/revista/v10/n3/pdf/v10n3a02.pdf

21. Johnson M, Bulechek G, Butcher H, Dochterman JM, Maas M, Moorhead S,et al. Ligações entre NANDA, NOC, NIC: diagnósticos, resultados e intervenções de enfermagem. Trad. RM Garcez. $2^{\text {a }}$ ed. Porto Alegre: Artmed; 2009.

22. Salvador PTCO, Oliveira RKM, Costa TD, Santos VEP, Tourinho FSV. Tecnologia e inovação para o cuidado em enfermagem. Rev. Enferm. UERJ. [Internet]. 2012 [cited 2013 out 16];20(1):111-7. Disponível em: http://www.facenf.uerj.br/v20n1/v20n1a19.pdf.

Artigo recebido em 13/10/2012.

Aprovado para publicação em 09/10/2013.

Artigo publicado em 31/12/2013. 